OPEN ACCESS

Edited by:

Roberta Lanzillo,

Federico II University Hospital, Italy

Reviewed by:

Jessica Frau,

ATS Sardegna, Italy

Ché Serguera,

Institut National de la Santé et de la

Recherche Médicale (INSERM), France

*Correspondence:

Natasa Giedraitiene

natasa.giedraitiene@gmail.com

Specialty section:

This article was submitted to

Multiple Sclerosis and

Neuroimmunology,

a section of the journal

Frontiers in Neurology

Received: 10 March 2021

Accepted: 14 May 2021

Published: 13 July 2021

Citation:

Giedraitiene N, Drukteiniene E, Kizlaitiene R, Cimbalas A, Asoklis $R$

and Kaubrys G (2021) Cognitive Decline in Multiple Sclerosis Is Related to the Progression of Retinal Atrophy and Presence of Oligoclonal Bands: A 5-Year Follow-Up Study.

Front. Neurol. 12:678735.

doi: 10.3389/fneur.2021.678735

\section{Cognitive Decline in Multiple Sclerosis Is Related to the Progression of Retinal Atrophy and Presence of Oligoclonal Bands: A 5-Year Follow-Up Study}

\author{
Natasa Giedraitiene ${ }^{1 *}$, Egle Drukteiniene ${ }^{2}$, Rasa Kizlaitiene ${ }^{1}$, Andrius Cimbalas ${ }^{2}$, \\ Rimvydas Asoklis ${ }^{2}$ and Gintaras Kaubrys ${ }^{1}$ \\ ${ }^{1}$ Center of Neurology, Clinic of Neurology and Neurosurgery, Institute of Clinical Medicine, Faculty of Medicine, Vilnius \\ University, Vilnius, Lithuania, ${ }^{2}$ Center of Eye Diseases, Clinic of Ear, Nose, Throat, and Eye Diseases, Institute of Clinical \\ Medicine, Faculty of Medicine, Vilnius University, Vilnius, Lithuania
}

Background: Brain atrophy, which is associated with cognitive impairment and retinal nerve fiber layer (RNFL) atrophy, is the main biomarker of neurodegeneration in multiple sclerosis (MS). However, data on the relationship between inflammatory markers, such as oligoclonal bands (OCBs) in the cerebrospinal fluid (CSF), and cognition, RNFL atrophy, and brain atrophy are scarce. The aim of this study was to assess the influence of RNFL thickness, brain atrophy markers, intrathecal OCBs, and the immunoglobulin G (lgG) index on cognitive decline over a 5-year period in patients with MS.

Methods: This prospective, single-center, observational cohort study included 49 patients with relapsing MS followed up over 5 years. At baseline, the patients underwent brain magnetic resonance imaging (MRI). Cognitive evaluation was performed using the Brief International Cognitive Assessment for MS (BICAMS), and RNFL thickness was assessed using optical coherence tomography (OCT). OCBs and lgG levels in the CSF were evaluated at baseline. The BICAMS, OCT, and MRI findings were re-evaluated after 5 years.

Results: A significant reduction in information processing speed, visual learning, temporal RNFL thickness, the Huckman index, and third ventricle mean diameter was found in all 49 patients with relapsing MS over the observation period $(p<0.05)$. Of the patients, 63.3\% had positive OCBs and 59.2\% had elevated IgG indices. The atrophy of the temporal segment and papillomacular bundle and the presence of OCBs were significantly related to a decline in information processing speed in these patients $(0<$ 0.05). However, brain atrophy markers were not found to be significant on the general linear models.

Conclusions: RNFL atrophy and the presence of OCBs were related to cognitive decline in patients with MS over a 5-year follow-up period, thereby suggesting their utility as potential biomarkers of cognitive decline in MS.

Keywords: multiple sclerosis, cognition, BICAMS, OCT, oligoclonal bands, brain atrophy 


\section{INTRODUCTION}

Multiple sclerosis (MS) is an inflammatory and neurodegenerative disease of the central nervous system (CNS) (1) that leads to demyelination and diffuse neurodegeneration in both the brain and spinal cord gray matter and white matter $(1,2)$. The course of the disease is usually relapsing-remitting from onset $(1,3)$. Studies have also shown the involvement of both inflammatory and neurodegenerative processes from the early stages of the disease $(2,4,5)$. However, it remains unknown whether early degeneration is an independent process in MS or whether it is secondary to inflammation $(2,5)$. Inflammation in MS is more obvious and can be easily assessed, documented, and monitored in patients. In contrast, neurodegeneration is more difficult to assess and monitor, especially in the early stages of the disease (5).

Understanding the mechanism and causes of neurodegeneration in MS may be fundamental to developing therapies that can help halt this process and presumably prevent the progression of disability $(2,3)$. Brain atrophy assessed using magnetic resonance imaging (MRI) may be a biomarker for early neurodegeneration and may help predict the prognosis and disease course. Nevertheless, the measurement of atrophy on MRI in routine clinical practice remains a hurdle $(6,7)$. The identification of sensitive and accessible markers of and diagnostic tools for neurodegeneration may help us understand the relationship between these markers and may facilitate the development of easy-to-use and low-cost tools for exploring the pathophysiology of neurodegeneration in MS (2-4).

Cognitive impairment in MS reflects the underlying inflammatory and neurodegenerative pathological features of the disease (8). It is present in up to $50-70 \%$ of patients with MS and significantly lowers their quality of life $(8,9)$. The most frequently observed cognitive problems include deficits in information processing speed, episodic memory, complex attention, and executive function $(8,10,11)$. The severity of cognitive impairment varies considerably among individuals and can be observed even in the early stages of the disease $(12,13)$. Brain imaging studies have demonstrated that cognitive impairment in MS is related to the loss of brain volume or brain atrophy, which is an important sign of neurodegeneration $(8,10)$. Cognitive impairment and brain atrophy have been classically considered as features that present in the advanced stages of the disease (14). However, numerous studies have demonstrated that both cognitive impairment and brain atrophy may occur in the early stages of the disease and even in clinically and radiologically isolated syndromes $(15,16)$.

Optical coherence tomography (OCT) measurements of the macular ganglion cell layer and retinal nerve fiber layer (RNFL) have been proposed as biomarkers of axonal damage in MS (17). Recently, retinal OCT has been used as a sensitive and practical

Abbreviations: OCB, oligoclonal band; BICAMS, Brief International Cognitive Assessment for Multiple Sclerosis; EDSS, Expanded Disability Status Scale; SDMT, Symbol Digit Modalities Test; BVMT-R, Brief Visuospatial Memory Test Revised; CVLT-II, California Verbal Learning Test, Second Edition; PMB, Papillomacular Bundle; BCR, Bicaudate Ratio. alternative to MRI for the evaluation of neurodegeneration in MS (17, 18). However, studies have demonstrated a strong relationship between cognitive impairment across various cognitive domains and RNFL atrophy even in patients without MS-related optic neuritis (19-21). Some studies also indicate that RNFL thickness and cognition could be sensitive biomarkers that can be used for discriminating relapsing and progressive forms of the disease $(21,22)$. RNFL thickness may be associated with brain atrophy and cognitive impairment; therefore, OCT may be useful in assessing CNS neurodegeneration in MS $(23,24)$.

The presence of oligoclonal bands (OCBs) in the cerebrospinal fluid (CSF) or an elevated immunoglobulin G (IgG) index in patients with MS supports the diagnosis. Persistent intrathecal inflammation, demonstrated by the presence of OCBs in the CSF (25), is one of the hallmarks of MS in up to $95 \%$ of patients $(25,26)$. Previous research demonstrated that the presence of CSF-OCBs in patients with MS tends to be related to widespread cognitive changes, especially worse visual memory (27) and larger periventricular lesion area on MRI (28). However, data on the relationship between inflammatory markers, such as CSF-OCBs or elevated IgG indices, and neurodegenerative markers, such as brain atrophy markers or RNFL thickness, in MS are limited (27).

The aim of this study was to assess the impact of neurodegenerative markers, such as RNFL thickness and brain atrophy, as well as inflammatory markers, such as intrathecal OCBs and the IgG index, on the cognitive decline in patients with MS over a 5-year follow-up period.

\section{MATERIALS AND METHODS}

This prospective, single-center, observational cohort study was conducted at Vilnius University Hospital Santaros Klinikos, Lithuania. Patients were enrolled and assessed between 2012 and 2019. All patients signed an informed consent form, and the study was approved by the appropriate institutional review board. The inclusion criteria were as follows: age between 18 and 60 years, presence of relapsing-remitting MS, and absence of relapse and/or steroid treatment at least 30 days before the enrollment assessment and during the follow-up assessment. All patients were on stable-disease-modifying therapy at least 3 months before the assessment, and none had a history of MS-associated optic neuritis. The exclusion criteria were the presence of primary or secondary progressive MS, neurological disorders other than MS, any vision or hearing problems that could influence performance on the tests, and optic neuritis during the observation period.

After providing signed written informed consent, all the patients underwent physical and neurological examinations, neuropsychological assessment using the Brief International Cognitive Assessment for MS (BICAMS), ophthalmological examination using OCT, and brain MRI. The same evaluations were repeated 5 years ( \pm 14 days) later. The changes from baseline to the follow-up visit were calculated for all assessments.

All patients with MS were diagnosed according to the McDonald criteria by a neurologist at the Vilnius Multiple Sclerosis Center $(29,30)$. Neurological disability was assessed 


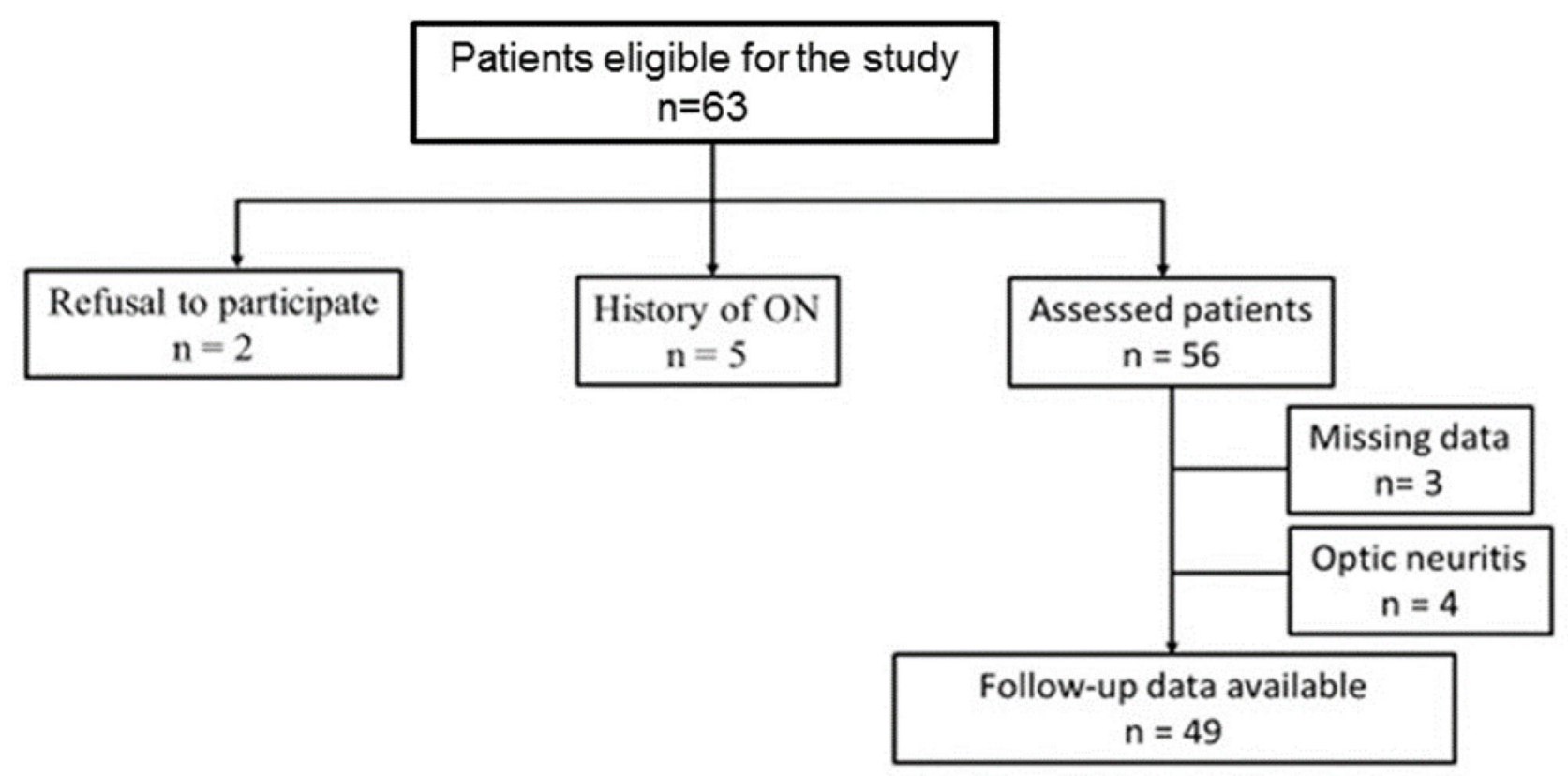

FIGURE 1 | Flowchart illustrating patient selection. ON, optic neuritis.

using the Expanded Disability Status Scale (EDSS) (31). The patients also underwent a lumbar puncture for evaluating the CSF-OCBs and IgG index at baseline.

\section{BICAMS}

All the patients were examined by the same neurologist, and the tests were administered in the same sequence: the Symbol Digit Modalities Test (SDMT) to evaluate the information processing speed; the Brief Visuospatial Memory Test Revised (BVMT$\mathrm{R})$, i.e., the first three recall trials to evaluate visual learning and memory; and the California Verbal Learning Test, Second Edition (CVLT-II), i.e., the first five trials to evaluate verbal learning and memory (32-35). The baseline and follow-up assessments were performed by the same neurologist. Different versions of the BICAMS test were used during the baseline and follow-up assessments.

\section{OCT}

OCT was performed on both eyes of each patient by using a spectral-domain OCT device (Spectralis, Heidelberg Engineering, Heidelberg, Germany), and the images were evaluated by the same ophthalmologist. RNFL thickness was measured using the RNFL-N axonal protocol with three 3.4mm-diameter circular scans. The RNFL Spectralis protocol generates maps with four quadrants (superior, inferior, nasal, and temporal) and six sector thicknesses (superonasal, nasal, inferonasal, inferotemporal, temporal, and superotemporal); it also measures the thickness of the papillomacular bundle (PMB), the nasal-to-temporal ratio, and the average thickness.

\section{MRI}

Brain MRI with gadolinium enhancement was performed in all patients by using a Siemens Aera 1.5 T MRI scanner (Siemens, Munich, Germany). MRI assessment included the following sequences: T1 (repetition time, $526 \mathrm{~ms}$; echo time, $14 \mathrm{~ms}$ ), T2 (repetition time, 4,110 ms; echo time, $105 \mathrm{~ms}$ ), and fluidattenuated inversion recovery (FLAIR) T2 (repetition time, $9,000 \mathrm{~ms}$; echo time, $122 \mathrm{~ms}$ ). A radiologist who was blinded to the patient's clinical data calculated the linear measures of brain atrophy. To evaluate brain atrophy, the Huckman index (sum of the greatest and smallest distances between the frontal horns), third ventricle width, and bicaudate ratio (BCR) were measured. The BCR was measured on a FLAIR axial image, where the heads of the caudate nuclei were best visible and closest to each other. The BCR was determined as the minimum intercaudate distance divided by the distance between the outermost parts of both the hemispheres measured along the same line.

\section{Statistical Analysis}

Data were analyzed using IBM SPSS Statistics for Windows, Version 23.1 (IBM Corp., Armonk, NY, USA). Continuous variables were reported as medians and ranges or means and standard deviations, while categorical variables were reported as absolute numbers and percentages of total patients. The normal distribution of the data was verified using the Shapiro-Wilk test. Student's $t$-test was used to compare the means between the two groups (baseline and follow-up assessments). The chi-square test was used for categorical variables. General linear regression was used to assess the relationship between the change in cognitive functions over 5 years (dependent variable) and the following clinical and demographic factors as explanatory variables: the 
TABLE 1 | Clinical and demographic characteristics of the patients.

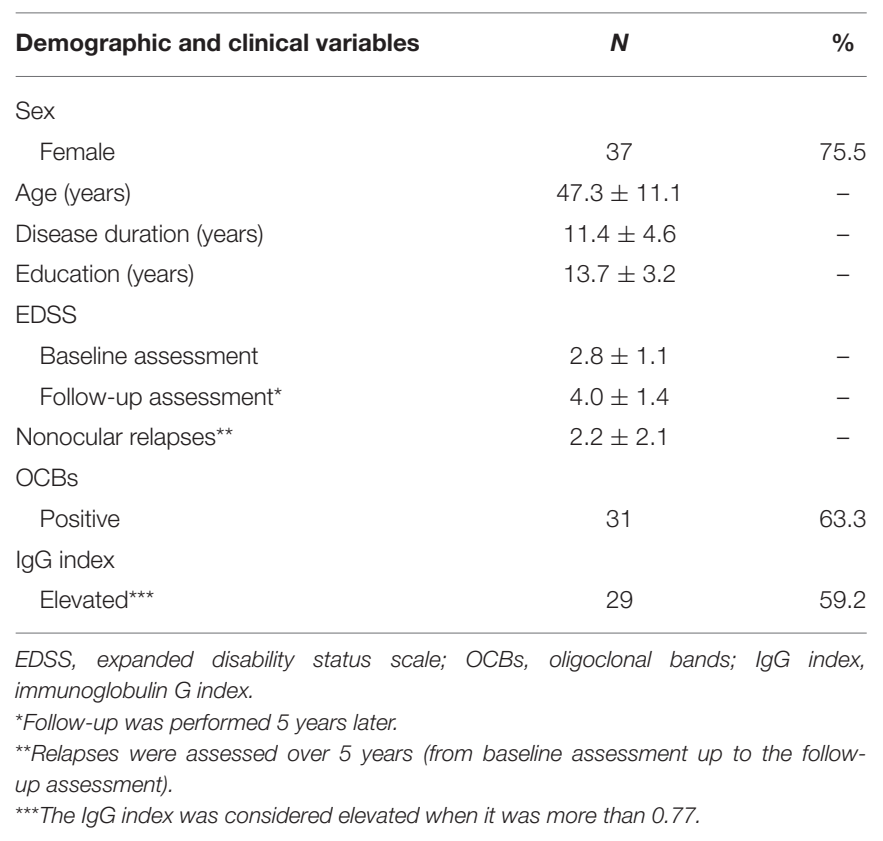

change in RNFL thickness over 5 years, the change in brain atrophy markers over 5 years, the presence of OCBs, the IgG index, disease duration, age, and sex. The dependent variables in the models were the changes in SDMT, BVMT-R, and CVLTII over 5 years. The independent variables (regressors) were the changes in different segments of the RNFL over 5 years in both eyes; the changes in brain atrophy markers on brain MRI (third ventricle width, Huckman index, or bicaudal score) over 5 years; the difference in the EDSS score between the baseline and followup assessments; and the IgG index, the presence of OCBs, age, sex, and disease duration at baseline. A value of $p<0.05$ was considered significant.

\section{RESULTS}

\section{Patients}

Sixty-three patients were enrolled in this study. The 5-year follow-up data were available for 49 patients (77.8\%) (Figure 1).

All patients had relapsing-remitting MS. The demographic and clinical characteristics of the patients are listed in Table $\mathbf{1 .}$

\section{CSF Assessment}

Of 49 patients, $63.3 \%$ had positive OCBs and $59.2 \%$ had elevated IgG indices in the CSF. Positive OCBs and elevated IgG indices did not differ according to sex $\left(\chi^{2}=0.079, p>0.05\right.$ and $\chi^{2}=$ $0.843, p>0.05$, respectively), age $(p>0.05)$, and disease duration $(p>0.05)$. The severity of disability was assessed using the EDSS at baseline, and the changes in EDSS scores between the baseline and follow-up assessments did not differ between patients with positive and negative OCBs $(p>0.05)$, as well as between patients with elevated and lower than normal IgG indices $(p>0.05)$. The incidence of positive OCBs and elevated IgG indices was similar in patients (63.3 and 59.2\%, respectively); however, no
TABLE 2 | Cognitive scores at baseline and follow-up in patients with MS.

\begin{tabular}{lccc}
\hline Test & Baseline assessment & Follow-up assessment* & $\boldsymbol{p}^{\boldsymbol{*}}$ \\
\hline SDMT & $44.5 \pm 12.6$ & $40.3 \pm 12.6$ & $\mathbf{2 0 . 0 0 1}$ \\
BVMT-R & $24.7 \pm 6.1$ & $23.1 \pm 7.2$ & $<\mathbf{0 . 0 5}$ \\
CVLT-II & $59.5 \pm 9.2$ & $57.4 \pm 11.5$ & $>0.05$
\end{tabular}

SDMT, symbol digit modalities test; BVMT-R, brief visuospatial memory test revised CVLT-II, california verbal learning test, Second Edition.

${ }^{*}$ Follow-up was performed 5 years after the baseline assessment.

**Student's t-test for paired samples. Bold values indicate significant differences or indicators.

relationship was found between positive OCBs and elevated IgG indices $\left(\chi^{2}=0.993, p>0.05\right)$.

\section{Cognitive Dynamics in Patients With MS}

The scores of the SDMT and BVMT-R were significantly lower during the follow-up assessment than at the baseline assessment, while the CVLT-II scores did not differ between the baseline and follow-up assessments (Table 2).

\section{RNFL Thickness Determined Using OCT}

The average RNFL thickness in the temporal, nasal, inferotemporal, and inferonasal segments and the overall global average thickness were significantly lower in both eyes at the follow-up assessment $(p<0.05)$, while the average thickness of the PMB was lower in the right eye and the thickness of the superotemporal segment was lower in the left eye. The OCT results are presented in Table 3.

\section{Linear Measures of Brain Atrophy}

The Huckman index and third ventricle width were significantly lower during the follow-up assessment than at the baseline assessment. However, the BCR did not differ between the baseline and follow-up assessments (Table 4).

\section{Relationship of Disease Characteristics and Biomarkers of Neurodegeneration and Inflammation to Cognitive Decline}

A general linear model was used to assess the relationship of the changes in RNFL thickness, brain atrophy markers, EDSS scores, OCBs, IgG index, and disease characteristics (age, sex, and disease duration) to the changes in cognitive domains over 5 years. The dependent variables in the models were the changes in SDMT, BVMT-R, and CVLT-II scores over 5 years. The independent variables (regressors) were the changes in different segments of the RNFL over 5 years, which were assessed as the changes in the mean values for both eyes; the changes in brain atrophy markers on brain MRI (third ventricle width, Huckman index, or bicaudal score) over 5 years; the differences in the EDSS scores between the baseline and follow-up assessments; and the IgG index, presence of OCBs, age, sex, and disease duration at baseline (Table 5). The decline in information processing speed over 5 years in patients with relapsing MS was explained by the RNFL thickness in the temporal segment or PMB in both eyes as well as the CSF-OCBs. 
TABLE 3 | Changes in RNFL thicknesses in patients with MS at the baseline and follow-up assessments.

\begin{tabular}{|c|c|c|c|c|c|c|}
\hline Segment & $\begin{array}{l}\text { Right eye, } \\
\Delta \text { RNFL }_{\mathrm{B}-5}{ }^{*} \\
\quad \pm \mathrm{SD}^{\star *}\end{array}$ & $p^{\star \star \star}$ & $\begin{array}{l}\text { Left eye, } \\
\begin{array}{l}\Delta \mathrm{RNFL}_{\mathrm{B}-5}{ }^{*} \\
\pm \mathrm{SD}^{\star *}\end{array}\end{array}$ & $p^{\star \star \star}$ & $\begin{array}{l}\text { Both eyes, } \\
\begin{array}{l}\Delta \text { RNFL }_{\mathrm{B}-5}{ }^{*} \\
\quad \pm \mathrm{SD}^{* *}\end{array}\end{array}$ & $p^{\star \star \star *}$ \\
\hline $\mathrm{T}$ & $2.2 \pm 4.1$ & $<0.001$ & $1.6 \pm 4.9$ & $<0.05$ & $1.9 \pm 3.7$ & $<0.001$ \\
\hline $\mathrm{N}$ & $4.2 \pm 4.9$ & $<0.001$ & $4.4 \pm 5.5$ & $<0.001$ & $4.3 \pm 4.3$ & $<0.001$ \\
\hline TS & $1.1 \pm 5.1$ & $>0.05$ & $1.8 \pm 5.9$ & $<0.05$ & $1.4 \pm 4.7$ & $<0.05$ \\
\hline $\mathrm{Tl}$ & $4.5 \pm 6.5$ & $<0.001$ & $3.9 \pm 7.4$ & $<0.001$ & $4.2 \pm 5.4$ & $<0.001$ \\
\hline NS & $-0.2 \pm 5.0$ & $>0.05$ & $0.9 \pm 6.0$ & $>0.05$ & $0.3 \pm 4.2$ & $>0.05$ \\
\hline $\mathrm{NI}$ & $4.3 \pm 6.8$ & $<0.001$ & $4.0 \pm 7.7$ & $<0.001$ & $4.2 \pm 5.6$ & $<0.001$ \\
\hline PMB & $1.9 \pm 3.6$ & $<0.001$ & $0.7 \pm 4.9$ & $>0.05$ & $1.3 \pm 3.3$ & $<0.05$ \\
\hline$G$ & $2.8 \pm 3.1$ & $<0.001$ & $2.7 \pm 4.4$ & $<0.001$ & $2.8 \pm 3.3$ & $<0.001$ \\
\hline
\end{tabular}

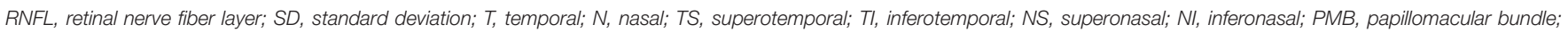
G, global.

${ }^{*}$ Change from the baseline to follow-up assessments: the mean of delta.

** Standard deviation of delta.

${ }^{* \star}$ Student's t-test for paired samples. Bold values indicate significant differences or indicators.

\section{DISCUSSION}

Cognitive impairment, RNFL thickness, and brain atrophy are markers of neurodegeneration in $\operatorname{MS}(21,29,36,37)$, whereas positive OCBs and elevated IgG indices in the CSF are markers of inflammation $(25,26)$. MRI was long considered the gold standard for monitoring the degenerative component of MS $(6,7)$. Thereafter, RNFL thickness and cognition were recognized as biomarkers of neurodegeneration $(14,17,18)$. The presence of CSF-OCBs in patients with MS is supportive of the diagnosis $(29,30)$, even though the relationship between the patient's clinical and cognitive features has not been thoroughly examined. In our study, positive OCBs were detected in $63.3 \%$ of patients and elevated IgG indices were detected in 59.2\%. CSF biomarkers such as OCBs and elevated IgG indices were not correlated with each other. Nevertheless, both are markers of inflammation and both are supportive of a diagnosis of MS (30). Previously published data regarding the correlation between the presence of OCBs and elevated IgG levels differ among studies; while some studies have reported positive correlations (38), others have not found any relationship (39-41). Moreover, in most patients with MS, when the number of OCBs is $>2$, no linear association is observed between CSF IgG levels and the number of OCBs $(39,40)$. The absence of such a correlation is possible because OCBs reflect the production of several monoclones, while the IgG index is a general indicator of enhanced autoimmune response. In our study, we did not find a correlation between the presence of OCBs and the IgG index.

We investigated whether cognitive decline over 5 years in patients with relapsing MS can be explained using neurodegenerative and inflammatory markers such as OCBs and IgG indices in the CSF. In the recently published revision of the McDonald diagnostic criteria, the detection of oligoclonal IgG bands in the CSF has regained importance (30). Therefore, we decided to assess the impact of inflammatory markers on cognitive decline. We found that among the
TABLE 4 | Brain atrophy markers at the baseline and follow-up assessments in patients with MS.

\begin{tabular}{lccc}
\hline $\begin{array}{l}\text { Brain atrophy } \\
\text { marker }\end{array}$ & $\begin{array}{c}\text { Baseline } \\
\text { assessment }\end{array}$ & $\begin{array}{c}\text { Follow-up } \\
\text { assessment }\end{array}$ & $\boldsymbol{p}^{\star *}$ \\
\hline HI & $49.3 \pm 7.3$ & $52.0 \pm 8.3$ & $<\mathbf{0 . 0 0 1}$ \\
TWW & $4.7 \pm 1.9$ & $6.3 \pm 2.1$ & $<\mathbf{0 . 0 0 1}$ \\
BCR & $0.1 \pm 0.03$ & $0.1 \pm 0.04$ & $>0.05$ \\
\hline
\end{tabular}

HI, huckman index; TVW, third ventricle width; BCR, bicaudate ratio.

${ }^{*}$ Follow-up was performed 5 years after the baseline assessment.

${ }^{*}$ Student's t-test for paired samples. Bold values indicate significant differences or indicators.

biomarkers of neurodegeneration and neuroinflammation, RNFL thickness in the temporal segment, PMB thickness in both eyes, and the presence of OCBs were explanatory variables indicating a decline in information processing speed in patients with MS.

Many studies have provided data on one particular cognitive measure, i.e., the SDMT, which is considered particularly sensitive to the decrease in information processing speed that is commonly seen in MS $(42,43)$. Owing to its high reliability, validity, sensitivity, and specificity, the SDMT has demonstrated superiority over other cognitive tests for MS in recent years (43). Our findings are consistent with previously published data (42-44), and the SDMT was the only cognitive test in which the results were related to other markers of neurodegeneration and inflammation in our cohort. Our data also confirmed the association between cognitive function and RNFL thickness. In particular, we found that the average thickness in the temporal segment and PMB in both eyes was the most important OCT measure related to cognitive decline in our patients. During the past decade, OCT has developed into a sensitive method for imaging neurodegeneration in MS (17, 45). Studies have demonstrated that lower average 
TABLE 5 | Regression models that explain the cognitive decline over 5 years in patients with MS.

\begin{tabular}{|c|c|c|c|}
\hline Dependent variable & Regression model & $R 2$ & $\begin{array}{c}p\left(R^{2} ;\right. \\
\text { coefficients) }\end{array}$ \\
\hline$\Delta \mathrm{SDMT}_{\mathrm{B}-5}$ & $-3.1-1.0 \times\left(\Delta \mathrm{RNFL}_{-} \mathrm{T}_{\mathrm{B}-5}\right)+3.3 \times$ CSF_OCBs & 0.599 & $<0.01$ \\
\hline$\Delta \mathrm{SDMT}_{\mathrm{B}-5}$ & $-8.8-1.1 \times\left(\Delta \mathrm{RNFL}_{-} \mathrm{PMB}_{\mathrm{B}-5}\right)+4.4 \times \mathrm{CSF}$ OCBs & 0.480 & $<0.01$ \\
\hline
\end{tabular}

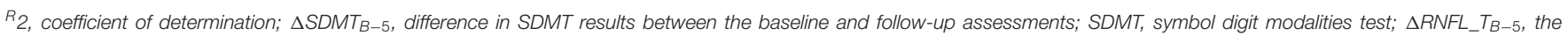

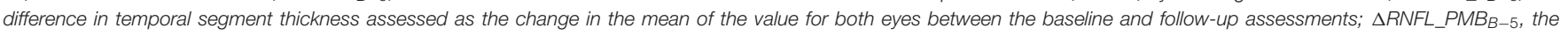

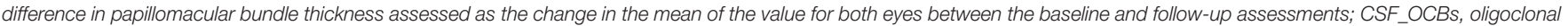
bands in the cerebrospinal fluid.

temporal RFNL thickness correlates with a more active disease course, higher EDSS at the time of assessment, and greater EDSS score increase over time $(37,46)$. Correlations were also found between RNFL thickness and performance on some tests of cognitive function in patients with MS, particularly the SDMT $(46,47)$. Our results are in line with these previously published data showing that the SDMT score and RNFL thickness in the temporal segment are significant cognitive and ophthalmological indicators of neurodegeneration in MS $(46,47)$.

The limitations of our study were the relatively small sample size and the lack of a control group. However, we did not identify any controlled study in which a comparison group was used to assess the presence of OCBs and IgG indices in the CSF or in which the patients were followed up for a long duration of 5 years.

Another innovative aspect of our work was the combined analysis of inflammatory OCBs, neurodegeneration-related RNFL thickness, and cognition. The dependence of cognition on the presence of OCBs and RNFL thickness has not been previously investigated. We found a relationship between both neurodegenerative and inflammatory markers and information processing speed. RNFL thickness in the temporal segment, PMB thickness, and the presence of OCBs could be considered biomarkers in the diagnostic workup for MS. We did not detect a significant influence of any other RNFL segment thickness or brain linear measurement on cognition in our cohort of patients with MS. Our results confirm that the BICAMS and OCT measure different aspects of neurodegeneration and that the thinning of the RNFL is a potential biomarker for cognitive disability in MS $(23,47)$, because we found that cognitive decline may be predicted not only by markers of degeneration but also by markers of intrathecal inflammation. These results imply that both the thinning of the RNFL and the presence of CSF-OCBs are feasible biomarkers for cognitive decline in MS.

\section{REFERENCES}

1. Klineova S, Lublin FD. Clinical course of multiple sclerosis. Cold Spring Harb Perspect Med. (2018) 8:a028928. doi: 10.1101/cshperspect.a0 28928

2. Lassmann H, Brück $\mathrm{W}$, Lucchinetti CF. The immunopathology of multiple sclerosis: an overview. Brain Pathol. (2007) 17:2108. doi: 10.1111/j.1750-3639.2007.00064.x

\section{DATA AVAILABILITY STATEMENT}

The original contributions presented in the study are included in the article/Supplementary Material, further inquiries can be directed to the corresponding author.

\section{ETHICS STATEMENT}

The studies involving human participants were reviewed and approved by The Lithuanian Bioethics Committee approved the study in 2011 (2011-01-27 No.: L-12-01/2), the permission to continue the study was granted by the Lithuanian Bioethics Committee in 2018 (2018-02-22 No.: 6B-18-41). The patients/participants provided their written informed consent to participate in this study.

\section{AUTHOR CONTRIBUTIONS}

NG contributed to the conception and design of the study, acquisition of the data, analysis and interpretation of the data, and drafting of the manuscript. ED contributed to the analysis and interpretation of the data and to the conception and design of the study. RK contributed to the conception and design of the study and the revision of the manuscript. AC contributed to the analysis and interpretation of the data and drafting of the manuscript. RA contributed to the analysis and interpretation of the data and drafting of the manuscript. GK contributed to the conception and design of the study, analysis and interpretation of the data, and drafting of the manuscript. All authors discussed the results and contributed to and approved the final manuscript.

\section{SUPPLEMENTARY MATERIAL}

The Supplementary Material for this article can be found online at: https://www.frontiersin.org/articles/10.3389/fneur. 2021.678735/full\#supplementary-material

3. Kamm CP, Uitdehaag BM, Polman CH. Multiple sclerosis: current knowledge and future outlook. Eur Neurol. (2014) 72:132-41. doi: 10.1159/000360528

4. Van Schependom J, Guldolf K, D'hooghe MB, Nagels G, D'haeseleer M. Detecting neurodegenerative pathology in multiple sclerosis before irreversible brain tissue loss sets in. Transl Neurodegener. (2019) 8:37. doi: 10.1186/s40035-019-0178-4

5. Chaudhuri A. Multiple sclerosis is primarily a neurodegenerative disease. $J$ Neural Transm (Vienna). (2013) 120:1463-6. doi: 10.1007/s00702-013-1080-3 
6. Rocca MA, Battaglini M, Benedict RH, De Stefano N, Geurts JJ, Henry RG, et al. Brain MRI atrophy quantification in MS: from methods to clinical application. Neurology. (2017). 88:403-13. doi: 10.1212/WNL.0000000000003542

7. De Stefano N, Airas L, Grigoriadis N, Mattle HP, O’Riordan J, Oreja-Guevara $\mathrm{C}$, et al. Clinical relevance of brain volume measures in multiple sclerosis. CNS Drugs. (2014) 28:147-56. doi: 10.1007/s40263-014-0140-z

8. Rocca MA, Amato MP, De Stefano N, Enzinger C, Geurts JJ, Penner IK, et al. Clinical and imaging assessment of cognitive dysfunction in multiple sclerosis. Lancet Neurol. (2015) 14:302-17. doi: 10.1016/S1474-4422(14)70250-9

9. Benedict RHB, Zivadinov R. Risk factors for and management of cognitive dysfunction in multiple sclerosis. Nat Rev Neurol. (2011) 7:33242. doi: 10.1038/nrneurol.2011.61

10. Chiaravalloti ND, DeLuca J. Cognitive impairment in multiple sclerosis. Lancet Neurol. (2008) 7:1139-51. doi: 10.1016/S1474-4422(08)70259-X

11. Oreja-Guevara C, Ayuso Blanco T, Brieva Ruiz L, Hernández Pérez MÁ, Meca-Lallana V, Ramió-Torrentà L. Cognitive dysfunctions and assessments in multiple sclerosis. Front Neurol. (2019) 10:581. doi: 10.3389/fneur.2019.00581

12. Feinstein A. Is there a cognitive signature for multiple sclerosisrelated fatigue? Mult Scler. (2015) 21:353-4. doi: 10.1177/13524585145 63099

13. Oset M, Stasiolek M, Matysiak M. Cognitive dysfunction in the early stages of multiple sclerosis-how much and how important? Curr Neurol Neurosci Rep. (2020) 20:22. doi: 10.1007/s11910-020-01045-3

14. Filippi M, Agosta F. Imaging biomarkers in multiple sclerosis. J Magn Reson Imaging. (2010) 31:770-88. doi: 10.1002/jmri.22102

15. Pérez-Miralles F, Sastre-Garriga J, Tintoré M, Arrambide G, Nos C, Perkal H, et al. Clinical impact of early brain atrophy in clinically isolated syndromes. Mult Scler. (2013) 19:1878-86. doi: 10.1177/13524585134 88231

16. Rojas JI, Patrucco L, Míguez J, Besada C, Cristiano E. Brain atrophy in radiologically isolated syndromes. J Neuroimaging. (2015) 25:6871. doi: 10.1111 /jon. 12182

17. Cruz-Herranz A, Balk LJ, Oberwahrenbrock T, Saidha S, Martinez-Lapiscina $\mathrm{EH}$, Lagreze WA, et al. The APOSTEL recommendations for reporting quantitative optical coherence tomography studies. Neurology. (2016) 86:2303-9. doi: 10.1212/WNL.0000000000002774

18. Coric D, Balk LJ, Verrijp M, Eijlers A, Schoonheim MM, Killestein J, et al. Cognitive impairment in patients with multiple sclerosis is associated with atrophy of the inner retinal layers. Mult Scler. (2018) 24:15866. doi: 10.1177/1352458517694090

19. Birkeldh U, Manouchehrinia A, Hietala MA, Hillert J, Olsson T, Piehl F, et al. Retinal nerve fiber layer thickness associates with cognitive impairment and physical disability in multiple sclerosis. Mult Scler Relat Disord. (2019) 36:101414. doi: 10.1016/j.msard.2019.101414

20. Ko F, Muthy ZA, Gallacher J, Sudlow C, Rees G, Yang Q, et al. Association of retinal nerve fiber layer thinning with current and future cognitive decline: a study using optical coherence tomography. Adv Biomed Res. (2015) 4:223. doi: 10.1001/jamaneurol.2018.1578

21. Bsteh G, Hegen H, Teuchner B, Amprosi M, Berek K, Ladstätteret F, et al. Peripapillary retinal nerve fibre layer as measured by optical coherence tomography is a prognostic biomarker not only for physical but also for cognitive disability progression in multiple sclerosis. Mult Scler. (2019) 25:196-203. doi: 10.1177/1352458517740216

22. Kizlaitiene R, Kaubrys G, Giedraitiene N, Ramanauskas N, Dementaviciene J. Composite marker of cognitive dysfunction and brain atrophy is highly accurate in discriminating between relapsing-remitting and secondary progressive multiple sclerosis. Med Sci Monit. (2017) 23:588-97. doi: 10.12659/MSM.903234

23. Frau J, Fenu G, Signori A, Coghe G, Lorefice L, Barracciu MA, et al. A cross-sectional and longitudinal study evaluating brain volumes, RNFL, and cognitive functions in MS patients and healthy controls. BMC Neurol. (2018) 18:67. doi: 10.1186/s12883-018-1065-9

24. Stellmann JP, Cetin H, Young KL, Hodecker S, Pöttgen J, Bittersohl D, et al. Pattern of gray matter volumes related to retinal thickness and its association with cognitive function in relapsing-remitting MS. Brain Behav. (2016) 7:e00614. doi: 10.1002/brb3.614
25. Villar LM, Masterman T, Casanova B, Gómez-Rial J, Espiño $M$, Sádaba MC, et al. CSF oligoclonal band patterns reveal disease heterogeneity in multiple sclerosis. J Neuroimmunol. (2009) 211:101-4. doi: 10.1016/j.jneuroim.2009.03.003

26. Dobson R, Ramagopalan S, Davis A, Giovannoni G. Cerebrospinal fluid oligoclonal bands in multiple sclerosis and clinically isolated syndromes: a meta-analysis of prevalence, prognosis and effect of latitude. J Neurol Neurosurg Psychiatry. (2013) 84:909-14. doi: 10.1136/jnnp-2012-304695

27. Anagnostouli M, Christidi F, Zalonis I, Nikolaou C, Lyrakos D, Triantafyllou $\mathrm{N}$, et al. Clinical and cognitive implications of cerebrospinal fluid oligoclonal bands in multiple sclerosis patients. Neurol Sci. (2015) 36:205360. doi: 10.1007/s10072-015-2303-1

28. Nakashima J, Fujihara K, Miyazawa H, Misu T, Fujimori J, Sato $\mathrm{S}$, et al. Relevance of callosal and periventricular MRI lesions to oligoclonal bands in multiple sclerosis. Acta Neurol Scand. (2006) 113:12531. doi: 10.1111/j.1600-0404.2005.00552.x

29. Polman CH, Reingold SC, Edan G, Filippi M, Hartung H-P, Kappos L, et al. Diagnostic criteria for multiple sclerosis: 2005 revisions to the "McDonald criteria." Ann Neurol. (2005) 58:840-6. doi: 10.1002/ana.20703

30. Thompson AJ, Reingold SC, Edan G, Filippi M, Hartung HP, Kappos L, et al. Diagnosis of multiple sclerosis: 2017 revisions of the McDonald criteria. Lancet Neurol. (2018) 17:162-73. doi: 10.1016/S1474-4422(17)30470-2

31. Kurtzke JF. Rating neurologic impairment in multiple sclerosis: an expanded disability status scale (EDSS). Neurology. (1983) 33:144452. doi: 10.1212/WNL.33.11.1444

32. Langdon DW, Amato MP, Boringa J, Brochet B, Foley F, Fredrikson $\mathrm{S}$, et al. Recommendations for a Brief International Cognitive Assessment for Multiple Sclerosis (BICAMS). Mult Scler. (2012) 18:891-8. doi: 10.1177/1352458511431076

33. Benedict RHB, Amato MP, Boringa J, Brochet B, Foley F, Fredrikson $\mathrm{S}$, et al. Brief International Cognitive Assessment for MS (BICAMS) international standards for validation. BMC Neurol. (2012) 12:55. doi: 10.1186/1471-2377-12-55

34. Giedraitiene N, Kaubrys G, Kizlaitiene R. Cognition during and after multiple sclerosis relapse as assessed with the brief international cognitive assessment for multiple sclerosis. Sci Rep. (2018) 8:8169. doi: 10.1038/s41598-018-26449-7

35. Giedraitiene N, Kizlaitiene R, Kaubrys G. The BICAMS battery for assessment of Lithuanian-speaking multiple sclerosis patients: relationship with age, education, disease disability, and duration. Med Sci Monit. (2015) 21:38539. doi: 10.12659/MSM.896571

36. Gordon-Lipkin E, Chodkowski B, Reich DS, Smith SA, Pulicken M, Balcer LJ, et al. Retinal nerve fiber layer is associated with brain atrophy in multiple sclerosis. Neurology. (2007) 69:1603-9. doi: 10.1212/01.wnl.0000295995.46586.ae

37. Sepulcre J, Murie-Fernandez M, Salinas-Alaman A, GarcíaLayana A, Bejarano B, Villoslada P. Diagnostic accuracy of retinal abnormalities in predicting disease activity in MS. Neurology. (2007) 68:1488-94. doi: 10.1212/01.wnl.0000260612.51849.ed

38. Simonsen CS, Flemmen HØ, Lauritzen T, Berg-Hansen P, Moen SM, Celius EG. The diagnostic value of IgG index versus oligoclonal bands in cerebrospinal fluid of patients with multiple sclerosis. Mult Scler J Exp Transl Clin. (2020) 6:2055217319901291. doi: 10.1177/2055217319901291

39. Beseler C, Vollmer T, Graner $\mathrm{M}$, Yu $\mathrm{X}$. The complex relationship between oligoclonal bands, lymphocytes in the cerebrospinal fluid, and immunoglobulin $G$ antibodies in multiple sclerosis: Indication of serum contribution. PLoS ONE. (2017) 12:e0186842. doi: 10.1371/journal.pone.0186842

40. Mares J, Herzig R, Urbanek K, Sladkova V, Sklenarova J, Bekarek V, et al. Correlation of the IgG index and oligoclonal bands in the cerebrospinal fluid of patients with multiple sclerosis. Biomed Pap Med Fac Univ Palacky Olomouc Czech Repub. (2008) 152:247-9. doi: 10.5507/bp.2008.038

41. Christensen O, Clausen J, Fog T. Relationships between abnormal IgG index, oligoclonal bands, acute phase reactants and some clinical data in multiple sclerosis. J Neurol. (1978) 218:237-44. doi: 10.1007/BF00 312879

42. Costa SL, Genova HM, DeLuca J, Chiaravalloti ND. Information processing speed in multiple sclerosis: Past, present, and future. Mult Scler. (2017) 23:772-89. doi: 10.1177/1352458516645869 
43. Benedict RHB, DeLuca J, Phillips G, LaRocca N, Hudson LD, Rudick $\mathrm{R}$, et al. Validity of the Symbol Digit Modalities Test as a cognition performance outcome measure for multiple sclerosis. Mult Scler. (2017) 23:721-33. doi: 10.1177/1352458517690821

44. Cohen JA, Reingold SC, Polman CH, Wolinsky JS; International Advisory Committee on Clinical Trials in Multiple Sclerosis. Disability outcome measures in multiple sclerosis clinical trials: current status and future prospects. Lancet Neurol. (2012). 11:467-76. doi: 10.1016/S1474-4422(12)70059-5

45. Costello F, Hodge W, Pan YI, Freedman M, DeMeulemeester C. Differences in retinal nerve fiber layer atrophy between multiple sclerosis subtypes. J Neurol Sci. (2009) 281:74-9. doi: 10.1016/j.jns.2009.02.354

46. Toledo J, Sepulcre J, Salinas-Alaman A, García-Layana A, Murie-Fernandez M, Bejarano B, et al. Retinal nerve fiber layer atrophy is associated with physical and cognitive disability in multiple sclerosis. Mult Scler. (2008) 14:906-12. doi: 10.1177/1352458508090221
47. Naseer MA, Fathi S, Roshdy NK, Labib DM, Khalil DH, Ibrahim W, et al. Cognitive and physical disability in Egyptian patients with multiple sclerosis: genetic and optical coherence tomography study. Neurol Res. (2019) 41:64451. doi: 10.1080/01616412.2019.1609203

Conflict of Interest: The authors declare that the research was conducted in the absence of any commercial or financial relationships that could be construed as a potential conflict of interest.

Copyright (c) 2021 Giedraitiene, Drukteiniene, Kizlaitiene, Cimbalas, Asoklis and Kaubrys. This is an open-access article distributed under the terms of the Creative Commons Attribution License (CC BY). The use, distribution or reproduction in other forums is permitted, provided the original author(s) and the copyright owner(s) are credited and that the original publication in this journal is cited, in accordance with accepted academic practice. No use, distribution or reproduction is permitted which does not comply with these terms. 